\title{
Géneros profesionales en la formación disciplinar: el caso de la licenciatura en Comunicación
}

\section{Professional genres in disciplinary training: the case of the Bachelor of Communication Degree}

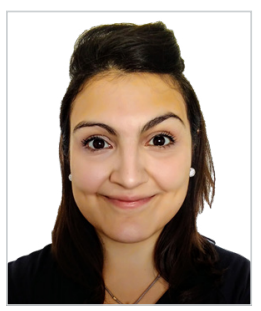

Antonela Georgina Dambrosio. Profesora y Licenciada en Letras por la Universidad Nacional del Sur (Bahía Blanca, Argentina). Es becaria doctoral del Consejo Nacional de Investigaciones Científicas y Técnicas (CONICET). Su tesis aborda el fenómeno de las fórmulas de tratamiento en las consignas de trabajo en distintos niveles educativos. Actualmente se desempeña como docente universitaria en el Departamento de Humanidades (UNS) y en la Universidad Salesiana (UNISAL). En esta última dirige, desde el año 2019, el proyecto "Géneros profesionales en la formación disciplinar: el caso de la Licenciatura en Comunicación".

Universidad Nacional del Sur-CONICET, Bahía Blanca, Argentina

antonela.dambrosio@uns.edu.ar

ORCID: 0000-0002-3780-8009

Recibido: 10/01/2020 - Aceptado: 08/02/2020

\section{Resumen:}

El proyecto de investigación que presentamos se propone aportar a un área no investigada en los estudios sobre géneros profesionales, centrando su atención en aquellos empleados en el desempeño profesional dentro del ámbito de la comunicación, a fin de generar propuestas de enseñanza acordes a las exigencias del campo laboral.

Palabras clave:

Géneros profesionales; comunicación; alfabetización especializada.

\section{Received: 10/01/2020 - Accepted: 08/02/2020}

\section{Abstract:}

The research project we present aims to contribute to a gap in professional genre studies. It focuses on those genres used in professional performance within the sphere of Communication Sciences to create teaching programmes according to the requirements of this specific field of work.

Keywords:

Professional genres; communication; specialized literacy.

\section{Presentación y objetivos del proyecto}

El abordaje de los géneros pertenecientes al ámbito académico cuenta con amplia tradición, tanto a nivel internacional como local. En este último caso, en los países de habla hispana, el estudio de un grupo de géneros afines -los géneros profesionales- ha atraído la atención de numerosos estudios en las últimas décadas. En este contexto, en su mayoría estos se han abocado al análisis de los géneros propios del desempeño profesional en áreas como la Medicina, la Ingeniería,

Cómo citar este artículo:

Dambrosio, A. G. (2020). Géneros profesionales en la formación disciplinar: el caso de la licenciatura en Comunicación. Doxa Comunicación, 30, pp. 167-175. 
las Ciencias Económicas y de la Administración, las Letras, Historia, entre otras, pero no así de la Comunicación en tanto campo profesional específico.

Si bien la revisión bibliográfica da cuenta de la existencia de trabajos que describen y analizan algunos de los géneros empleados por los comunicadores, ellos están elaborados en gran parte desde el ámbito del Periodismo, y, por lo tanto, no agotan la vasta diversidad de textos escritos y orales propios del desempeño profesional de un licenciado en Comunicación.

El proyecto que presentamos se desarrolla en la Universidad Salesiana (UNISAL) cuya sede se sitúa en Bahía Blanca (Argentina) y constituye la única institución en la ciudad cuya oferta académica incluye la carrera de Licenciatura en Comunicación. El proyecto cuenta actualmente con financiación por parte de la Secretaría de Investigación de la Universidad (RES. CS. N04/2019).

Tal como señalan investigaciones como las de Intriago Macías y Quevedo Arnaiz (2016) para el caso concreto de la Comunicación Social, en los últimos años los modelos educativos -aunque no siempre formalmente desde sus planes de estudios- se han centrado en el fomento del desarrollo de distintas competencias en el campo profesional, competencias que van más allá de los contenidos disciplinares adquiridos a lo largo de la formación. Entre ellas, podemos situar las prácticas de lectura, escritura y oralidad. En este sentido, el propósito rector de este proyecto de investigación es abordar la problemática de los géneros profesionales circunscripta en un caso concreto -el de la Licenciatura en Comunicación-a fin de diseñar un corpus representativo de los textos orales y escritos que los egresados deberán comprender y producir a lo largo de su desempeño profesional.

La relevancia de esta tarea reside en que es precisamente el dominio de los textos de una comunidad discursiva lo que permite que los estudiantes comiencen a formar parte de ella (Cassany, 2010). Debido a que son los textos los que -siguiendo a Bazerman y Paradis (1991)- mantienen en gran parte la organización, poder y actividad de las profesiones, nuestro foco de interés está puesto en aquellos que aún no han sido estudiados o los que lo han sido en menor medida pero que también forman parte de las tareas profesionales cotidianas de un trabajador de la comunicación. Tal es el caso, por ejemplo, de los géneros de modalidad oral producidos interprofesionalmente y entre profesionales y miembros no expertos de la comunidad.

En una sociedad cada vez más especializada, en donde se exige a los profesionales un dominio eficaz de los discursos que genera su actividad, es necesaria una atención particular a estas formas de comunicación (López Ferrero, 2002: 196). A fin de poder afianzar estas competencias y formar profesionales preparados para el campo laboral que los aguarda, resulta de vital importancia lograr un conocimiento descriptivo acerca del material que se produce, no solo en el ámbito de la universidad, sino principalmente en la vida laboral cotidiana de los profesionales (Parodi, 2015: 77).

En sus estudios, Parodi hace hincapié en la necesidad de contar con investigaciones "basadas en corpus de textos amplios, ecológicos y diversificados" (Parodi y Burdiles, 2015: 32) en lo que respecta al estudio de los géneros académicos y profesionales, fundamentalmente porque solo así se logrará conformar una base empírica sólida sobre la que construir una alfabetización especializada (Parodi, 2015), fin último de esta investigación. 
Tal como se anticipó, el objetivo general del presente proyecto es contribuir al fortalecimiento de las competencias profesionales de escritura y oralidad en la carrera de la Licenciatura en Comunicación.

Entre sus propósitos específicos se encuentran: confeccionar un corpus representativo de los géneros propios del campo profesional de un licenciado en Comunicación y promover la construcción de herramientas de enseñanza y evaluación desde las distintas cátedras acordes a las exigencias del campo laboral y al perfil del comunicador formado en la UNISAL.

\section{Antecedentes}

En lo que respecta a los estudios en torno a los géneros académicos y profesionales, a lo largo de su trayectoria, estos se han centrado prioritariamente en la producción escrita de distintas disciplinas. Las primeras investigaciones fueron desarrolladas en el ámbito anglosajón de la mano de Swales (1990), Bazerman y Paradis (1991), Bathia (2013) y Trosborg (2000). Por su parte, en España destacan los aportes de López Ferrero (2002) y Cassany y López Ferrero (2010) en torno a las prácticas letradas profesionales y la necesidad de la preparación universitaria a fin de fomentar recursos y habilidades que permitan a los alumnos apropiarse de las prácticas profesionales de manera eficaz. En América del Sur, particularmente en Chile, destacamos las investigaciones desarrolladas por Parodi $(2008,2010)$ y Parodi y Burdiles (2015) en torno al género manual y otros textos disciplinares de diversas áreas. En Argentina, son insoslayables los aportes de Ciapuscio (1994) en torno a los tipos textuales y la definición, caracterización y clasificación de textos especializados como así también los de Cubo de Severino (2005) y su grupo de investigación, reconocidos internacionalmente por sus estudios sobre los géneros propios del discurso académico y científico. El proyecto dirigido por Bosio en la Universidad Nacional de Cuyo continúa esta línea y recientemente incorpora a su corpus eventos comunicativos orales enmarcados dentro del ámbito académico. Por su parte, Navarro ha realizado numerosos trabajos en los que aborda la enseñanza de los géneros profesionales en la universidad en las áreas de Economía y Administración $(2010,2018)$ como también la problemática en sí del abordaje de los discursos profesionales (2012) y la alfabetización disciplinar (2017). Finalmente, en el ámbito local bahiense, destacamos los estudios desarrollados por Vallejos (2000 y 2004) y Castro Fox y Vallejos (2013) en torno al discurso científico pedagógico.

Los géneros profesionales han sido objeto de reflexión también dentro de las investigaciones en torno a la comunicación en el lugar de trabajo y el discurso institucional. Entre ellas, distinguimos las de Mumby y Clair (2001) y de Gunnarson (2001). Desde una perspectiva de análisis de corte sociolingüístico, Holmes y Stubbe (2003) analizan distintas estrategias de cortesía que tienen lugar en la comunicación laboral, mientras que Arminen (2005), Heritage y Clayman (2010), Pickering, Frinigal y Staples (2016) abordan la interacción profesional en diversos dominios. En el marco de los estudios realizados en Latinoamérica, el volumen coordinado por Rebeil Corella y Ruiz Sandoval Reséndiz (1998) en el que se abordan distintos aspectos relacionados con la comunicación en las empresas constituye un importante aporte. En nuestra variedad dialectal -el español bonaerense- destacan los trabajos de Julián (2015, entre otros) en torno a las manifestaciones de (des)cortesía en puestos de atención al público en instituciones de la ciudad de Bahía Blanca. 
También en el marco de estudios del español como lengua de especialidad, se analizan algunos géneros profesionales. En este sentido, resultan relevantes los aportes de Gómez de Enterría Sánchez (2009) en relación con la comunicación escrita y oral en la empresa.

Por último, si bien dentro del campo específico de la investigación en el área de Comunicación no se han registrado trabajos en los que se aborde específicamente la problemática de los géneros propios de la disciplina, nos interesa recuperar el estudio de Amado (2010) acerca de la prensa, la gestión de la comunicación en las organizaciones y la generación de información.

La faceta profesional de la labor del comunicador ha sido estudiada, por un lado, en los trabajos de Intriago Macías y Quevedo Arnaiz (2016) y Juárez (2017) en torno a la identidad profesional del comunicador; y por otro, en los de Vásquez Donoso, Marroquin Velásquez y Ángel Botero (2018) y Zarowsky y Justo von Lurzer (2018) quienes esbozan los principales líneas que ha seguido la investigación en Comunicación en América Latina y, concretamente, en Argentina.

La breve reseña trazada pone de manifiesto que en las investigaciones realizadas hasta el momento el foco ha estado puesto en los géneros profesionales escritos y que no existe -hasta donde llega nuestro conocimiento- una propuesta de la índole de la que presentamos, que se aboque al diseño de un corpus de textos (orales y escritos) propios del campo disciplinar de la Licenciatura en Comunicación.

\section{Marco teórico}

El contexto conceptual del presente estudio, como también su marco metodológico, responde a un diseño flexible, habida cuenta de la posibilidad de que en el proceso de investigación puedan surgir situaciones nuevas e inesperadas vinculadas con el tema de estudio que puedan implicar cambios en las hipótesis y propósitos, como así también la incorporación de otras técnicas de recolección y procesamiento de datos (Vasilachis, 2006: 67).

La investigación se desarrolla desde un enfoque pragmático-sociocognitivo (Heinemann y Vieghweger, 1991; Bathia, 2014; Parodi, 2010, 2015; Parodi y Burdiles, 2015). Este propone una aproximación multinivel de los géneros profesionales, ya que una clasificación que considere un único criterio no permite dar cuenta de la complejidad y dinamismo de los rasgos que los caracterizan.

En nuestra concepción de género nos adscribimos a los planteamientos propuestos por Bathia (2013, 2014), Cassany (2006), Parodi (2015) y Navarro (2012). En este sentido, entendemos los géneros como constructos cognitivos que se articulan de modo integral en tres dimensiones: cognitiva, social y lingüística. Estos constituyen constelaciones de potencialidades de convenciones discursivas, sustentadas por los conocimientos previos de los hablantes y oyentes a partir de constricciones y parámetros contextuales, sociales y cognitivos (Parodi, 2015: 26). En su manifestación concreta, son variedades de una lengua que operan a través de conjuntos de rasgos lingüístico-textuales co-ocurrentes sistemáticamente a través de las tramas de un texto y que se circunscriben lingüísticamente en virtud de distintos factores tales como los propósitos comunicativos, participantes implicados, ámbitos de uso, soportes y medios, entre otros (ibídem). 
En este contexto, Parodi ubica a los géneros profesionales dentro del discurso especializado junto con los géneros académicos, y ambos conforman un continuum que permite articular el mundo del aprendizaje con el de la práctica profesional concreta. Asimismo, y si bien prefiere la denominación de discurso profesional, compartimos la concepción de Navarro (2012) de este objeto, y consideramos los géneros profesionales como "el conjunto de géneros discursivos que llevan a cabo los objetivos específicos de las organizaciones donde circulan. Estos constituyen prácticas discursivas que se encuentran estandarizadas y reguladas institucionalmente" (2012: 1297-1298).

Por su parte, dada la índole de los contextos de producción y circulación de los géneros objeto de estudio -y sobre todo los de modalidad oral-, la dimensión interaccional constituirá uno de los principales ejes de análisis ya que, como señala Gómez de Enterría Sánchez, esta “contribuirá poderosamente a facilitarnos todos los significados extralingüísticos que se ponen en práctica durante el intercambio comunicativo, así como toda la simbología que surge de la relación entre lengua y cultura dentro de cada ámbito de especialidad" (2009: 130). En este sentido es que adscribimos a los planteamientos de la Sociolingüística Interaccional (Gumperz, 1982 y 2001; Tannen, 2004), de la Pragmática Sociocultural (Bravo y Briz, 2004) y que incorporamos nociones teóricas correspondientes al Análisis del Discurso Institucional (Prego Vázquez, 1998; Drew y Sorjonen, 2001; Benwell y Stokoe, 2006; Heritage y Clayman, 2010).

\section{Consideraciones metodológicas}

López Ferrero sostiene que "para dar cuenta de la complejidad de la construcción y la interpretación de los géneros producidos en contextos profesionales, es necesario establecer puentes entre distintas metodologías" (2002: 195). Así, de acuerdo con el marco teórico señalado, se trabaja con los planteamientos metodológicos de los modelos multinivel referidos anteriormente a la vez que con técnicas de recolección y procesamiento de datos propias de la Sociolingüística Interaccional y la Pragmática Sociocultural.

Para la conformación del corpus de análisis se trabaja con una combinación de técnicas de recolección de datos:

- Técnica de participante observador en diversos ámbitos en los que se desempeñen profesionales del área de Comunicación: empresas, organizaciones estatales y administrativas, instituciones comerciales, medios de comunicación, sociedades de fomento, agrupaciones juveniles, entre otras.

- Entrevistas semiestructuradas a profesionales que ejerzan roles de comunicación en el marco de los dominios señalados anteriormente.

- Análisis de fuentes: textos elaborados por estos profesionales dirigidos tanto a otros de diferentes disciplinas como a miembros de la comunidad en general (a nivel interno y externo de las instituciones); manuales elaborados por consultoras, entre otros.

Es importante destacar, como también sostiene Navarro (2012), que aspiramos al trabajo asociado entre los investigadores y los escritores de la comunidad profesional abordada.

Los textos recabados a lo largo del trabajo de campo pretenden conformar una muestra de lengua real y por este motivo no son ni mutilados ni adaptados. 
Para el procesamiento de los datos y el posterior diseño del corpus con sus respectivas clasificaciones se vuelve necesaria la integración de herramientas básicas del área de la Lingüística de Corpus (Wynne, 2004; O’Keeffe y McCarthy, 2010) y los planteamientos realizados en este sentido para el análisis de discursos especializados por Parodi (2007). Asimismo, se incorporan aportes de las propuestas esbozadas en Bosio y Sacerdote (2018) y Bosio, Castro y Cubo (2018).

Los parámetros que se toman en cuenta tanto para la recolección de datos como para el procesamiento y diseño del corpus incluyen características internas de los textos como también el contexto extralingüístico en el que estos de producen y circulan. A continuación, los presentamos en un listado tentativo, sujeto a futuras revisiones en función de lo recabado en la labor de trabajo de campo.

- Aspectos contextuales y situacionales: (macro)propósito comunicativo o función; ámbito de circulación (mediático, empresarial, administrativo, comercial, estatal); coordenadas espacio-temporales (comunicación diferida, mediada o cara a cara); soporte; participantes involucrados (características sociales -edad, género, lugar de nacimiento, lengua materna- ; nivel de experticia -legos o no legos)-; posición ocupada-director, gerente, empleado-; roles sociales -simétricos/asimétricos, cercanos/lejanos-); historia del género.

- Aspectos discursivos: información contenida y desarrollo; organización de la información; intensificación o ponderación, atenuación; modalidad; máximas comunicativas específicas.

- Aspectos léxico-gramaticales: empleo de terminología específica; estructuras sintácticas que lo caracterizan; uso particular de la puntuación; potencial cambio de código o uso de préstamos léxicos.

En lo que respecta a las estrategias de acceso a las distintas instituciones en las que se realiza el trabajo de campo, se solicitan las respectivas autorizaciones a los directivos y personas a cargo en entrevistas personales y pedidos formales, a la vez que se requiere la firma de consentimientos informados por parte de los profesionales que decidan participar de la investigación. En todos los casos se emplean distintas técnicas de anonimización a fin de preservar las identidades de estos (Sampson, 2000; De- Matteis, 2014; Cantamutto y Vela Delfa, 2015).

El principal aporte de la propuesta radica en la incorporación de géneros poco explorados, ya sea porque su contexto de producción no ha sido objeto de interés en las investigaciones -tal como podría ser el caso de los géneros producidos en el marco de asociaciones barriales-, por dificultades de acceso (como puede ocurrir con textos producidos al interior de algunas empresas) o porque la atención de este tipo de abordajes ha estado mayoritariamente ligada al ámbito escrito, relegando así, géneros de modalidad oral.

\section{Referencias bibliográficas}

Amado, A. (2010). Prensa y comunicación. Relaciones informativas responsables, Buenos Aires: La Crujía.

Arminem, I. (2005). Institutional Interaction. Studies of Talk at Work, Surrey: Ashgate Publishing Company.

Bathia, V. K. (2013) [1993]. Analysing genre, London \& New York: Routledge.

Bathia, V. K. (2014) [2004]. Worlds of written discourse. A genre-based view, London, New York: Bloomsbury. 
Bazerman, Ch. y J. Paradis (eds.) (1991). Textual Dynamics of the Professions. Historical and contemporary studies of writing in professional communities, Wisconsin: University of Wisconsin Press.

Benwell, B. y E. Stokoe (2006). Institutional identities. Discourse and identity, Edinburgh: Edinburgh University Press, pp. 87-128.

Bosio, I. V. y Sacerdote, C. A. (2018). La construcción del Corpus EspaDA-UNCuyo. Objetivos y proyecciones. CHIMERA. Romance Corpora and Linguistic Studies, 5.2, pp. 79-85. (DOI: http://dx.doi.org/10.15366/chimera2018.5.2.007).

Bosio, I. V., Castro, C. y Cubo, L. (2018). Corpus EspaDA-UNCuyo. Revisión y discusión de criterios externos para la sistematización de géneros académicos orales. CHIMERA. Romance Corpora and Linguistic Studies, 5.2, pp. 87-94. (DOI: http://dx.doi.org/10.1536 6/chimera2018.5.2.008).

Bravo, D. y Briz, A. (eds.) (2004). Pragmática sociocultural: estudios sobre el discurso de cortesía en español, Barcelona: Ariel Lingüística.

Cantamutto, L. y Vela Delfa, C. (2015). Problemas de recogida y fijación de muestras del discurso digital. CHIMERA. Romance Corpora and Linguistic Studies, 2, pp. 131-155. (https://revistas.uam.es/index.php/chimera/article/view/1189)

Cassany, D. (2006). Taller de textos. Leer, escribir y comentar en el aula, Barcelona: Paidós.

Cassany, D. y López Ferrero, C. (2010). De la Universidad al mundo laboral: Continuidad y contraste entre las prácticas letradas académicas y profesionales. En Parodi, G. (ed.). Alfabetización académica y profesional en el siglo XXI: leer y escribir desde las disciplinas. Barcelona: Planeta Ariel, pp. 347-374.

Castro Fox, G. y Vallejos, P. (2013). Textos universitarios. Claves de lectura y producción, Bahía Blanca, EdiUNS.

Ciapuscio, G. E. (1994). Tipos textuales, Buenos Aires: Eudeba.

Cubo de Severino, L. (coord.) (2005). Los textos de la ciencia. Principales clases del discurso académico científico, Córdoba: Comunicarte.

De-Matteis, L. M. A. (2014). Ejes para un debate sobre el uso ético de datos interaccionales escritos y orales obtenidos en línea. Actas de las I Jornadas de Humanidades Digitales. Las humanidades digitales desde Argentina. Tecnologías, culturas, saberes, Buenos Aires: FFyL, UBA, pp. 235-247. (http://repositoriodigital.uns. edu.ar/bitstream/123456789 /2851/1/DeMatteis\%2C\%20L.\%20Ejes\%20para\%20un\%20debate....pdf)

Drew P. y Sorjonen, M. L. (2001). Diálogo institucional. En Van Dijk, T. (comp.), El discurso como interacción social. Estudios sobre el discurso II. Una introducción multidisciplinaria, Barcelona: Gedisa Editorial, pp. 141-178.

Gómez de Enterría, Sánchez, J. (2009). El español lengua de especialidad: enseñanza y aprendizaje, Madrid: Arco Libros.

Gumperz, J. (1982). Discourse strategies, Cambridge: Cambridge University Press.

Gumperz, J. (2001). Interactional Sociolinguistics: a Personal Perspective. En Schiffrin et al. (eds.) The Handbook of Discourse Analysis, Massachusetts: Blackwell Publishers, pp. 215-228.

Gunnarson, B. L. (2001). Análisis aplicado del discurso. En Van Dijk, T. (comp.), El discurso como interacción social. Estudios sobre el discurso II. Una introducción multidisciplinaria, Barcelona: Gedisa Editorial, pp. 405-441. 
Heinemann, W. y Viehweger, D. (1991). Textlinguistik. Eine Einführung, Tübingen, Niemeyer.

Heritage, J. y Claymanm, S. (2010). Talk in action. Interactions, Identities and Institutions, Oxford: Wiley-Blackwell.

Holmes, J. y Stubbe, M. (2003). Power and politeness in the workplace. A sociolinguistic analysis of talk at work, Abingdon y New York: Routledge.

Intriago Macías, C. y Quevedo Arnaiz, N. (2016). Competencias comunicativas profesionales en la formación del comunicador social. Mikarimin. Revista científica multidisciplinaria, Vol.2, №2, pp. 43-52. (http://45.238.216.13/ojs/index.php/ mikarimin /article/view/340)

Juárez, A. (2017). Análisis de las regulaciones académicas en la formación de los Comunicadores Sociales en las Instituciones Universitarias Argentinas en el contexto social actual. Estudio de caso. Argonautas, Año 7, n 9, pp.1-22. (http:// fchportaldigital. unsl.edu.ar/index.php/ARGO/article/download/11/11)

Julián, G. (2015). Interacción comunicativa en español bonaerense: manifestaciones y percepciones de la (des)cortesía en puestos de atención al público en instituciones, Tesis Doctoral, Bahía Blanca, Departamento de Humanidades, UNS.

López Ferrero, C. (2002). Aproximación al análisis de los discursos profesionales. Revista Signos, v.35, n 51-52, pp. 195215. (DOI: http://dx.doi.org/10.4067/S0718-09342002005100013)

Mumby, D. K. y Clair, R. P. (2001). El discurso de las organizaciones. en Van Dijk, T. (comp.), El discurso como interacción social. Estudios sobre el discurso II. Una introducción multidisciplinaria, Barcelona: Gedisa Editorial, pp. 263-296.

Navarro, F. (2010). La enseñanza de géneros profesionales El caso del plan de negocios en carreras de economía. Libro de Actas del Congreso Regional de la Cátedra UNESCO en Lectura y Escritura. Cultura Escrita y Políticas Pedagógicas en las Sociedades Latinoamericanas Actuales, Universidad Nacional de General Sarmiento, pp. 1520-1533.

Navarro, F. (2012). ¿Qué son los géneros profesionales? Apuntes teórico-metodológicos para el estudio del discurso profesional. En A. Cristófalo y J. Ledesma (Eds.), Actas del IV Congreso Internacional de Letras "Transformaciones CultuChiodiales. Debates de la teoría, la crítica y la lingüística en el Bicentenario", Buenos Aires: Universidad de Buenos Aires, pp. 1294-1303. (http://eventosacademicos.filo.uba.ar/index.php/CIL/IV-2010/paper/viewFile/2813/1231)

Navarro, F. (2017). De la alfabetización académica a la alfabetización disciplinar. En Ibáñez, R. y González, C. (eds.). Alfabetización disciplinar en la formación inicial docente. Leer y escribir para aprender, Valparaíso: Ediciones Universitarias de Valparaíso, pp. 7-15. (https://www.academia.edu/35528029/De_la_alfabetizaci\%C3\% B3nacad\%C3\%A9micaalaalfabetizaci\%C3\%B3n_disciplinar).

Navarro, F. y Mari, L. A. (coords.) (2018). Manual de lectura, escritura y oralidad en economía y administración, Bernal: Universidad Nacional de Quilmes.

O'Keeffe, A. y Mc Carthy, M. (2010). The Routledge Handbook of Corpus Linguistics, Abingdon: Routledge.

Parodi, G. (ed.) (2007). Lingüistica de Corpus y Discursos Especializados: puntos de mira, Valparaíso: Ediciones Universitarias de Valparaíso. 
Parodi, G. (2010). Academic and Professional Discourse Genres in Spanish, Amsterdam/Philadelphia: John Benjamins Publishing Company.

Parodi, G. (ed.) (2015) [2008]. Géneros académicos y géneros profesionales: accesos discursivos para saber y hacer, Valparaíso: Ediciones Universitarias de Valparaíso.

Parodi, G. y Burdiles, G. (eds.) (2015). Leer y escribir en contextos académicos y profesionales: géneros, corpus y métodos, Santiago de Chile: Editorial Planeta Chilena.

Pickering, L., Frinigal, E. y Staples, Sh. (2016). Talking at work. Corpus based explorations of Workplace Discourse, London: Palgrave Macmillan.

Prego Vázquez, G. (1998). Algunas consideraciones sobre el discurso institucional. Interlingüística, №9, pp. 265-268. (https://dialnet.unirioja.es/servlet/articulo?codigo=89 7083).

Rebeil Corella, M. A. y Ruiz Sandoval Reséndiz, C. (1998). El poder de la comunicación en las organizaciones, México: Plaza y Valdés.

Sampson, G. (2000). CHRISTINE Corpus: Documentation. (https://www.grsampson. net/ChrisDoc.html).

Swales, J. (1990). Genre Analysis. English in academic and research settings, Glasgow: Cambridge University Press.

Tannen, D. (2004). Interactional Sociolinguistics. En Ammon, U. et al., Sociolinguistics. An International Handbook of the Science of Language and Society, Vol. 1, Berlin and New York: Walter de Gruyter, pp. 76-88.

Trosborg, A. (ed.) (2000). Analysing profesional genres, Ámsterdam/Philadelphia: John Benjamins Publishing Company.

Vallejos, P. (2000). Prácticas discursivas en la producción del conocimiento científico, Bahía Blanca: Universidad Nacional del Sur.

Vallejos, P. (2004). El discurso científico pedagógico. Aspectos de la textualización del "saber enseñado", Bahía Blanca: EdiUNS.

Vasilachis de Gialdino, I. (coord.) (2006). Estrategias de investigación cualitativa, Barcelona: Gedisa Editorial.

Vásquez Donoso, C. Marroquín Velásquez, L. Ángel Botero, A. (2018). En busca de un enfoque latinoamericano de la comunicación organizacional: una revisión crítica de la producción académica de 2010 a 2014. Communication Theory, Vol. 28, Iss. 2, pp.155-179. (DOI: https://doi.org/10.1093/ct/qtx010).

Wynne, M. (ed.) (2004). Developing Linguistic Corpora: a Guide to Good Practice, Osbow Books.

Zarowsky, M. y Justo von Lurzer, C. (2018). Investigación en comunicación en la Argentina (2001-2015): entre la expansión y la intervención intelectual. Communication Theory, Vol.28, Iss. 2, pp. 202-223. (DOI: https://doi.org/10.1093/ct/qtx012). 